\title{
OPTICAL COHERENCE TOMOGRAPHY ANGIOGRAPHY: NEW HORIZON
}

\author{
Mostafa, E. \\ Ophthalmology dept., Faculty of Medicine, Sohag Univ., Sohag, Egypt \\ E-mail: engymostafa@yahoo.com
}

Received: $12 / 10 / 2021$

Accepted: $1 / 12 / 2021$

\section{Introduction}

Since 2014, Optical Coherence Tomography Angiography (OCT-A) has become available commercially [1]. With the use of this evolving technology, it is now possible to observe retinal vasculature in vivo. This was not applicable using the two milestones in retinal

\section{How Does OCT-A Work?}

OCT-A is considered an upgrade to OCT. The new technology uses motion contrast to detect vessels. The concept depends on the fact that stationary objects will not change when two successive images of it are taken. Yet, moving objects will become apparent. OCTA captures A-scans at the same

\subsection{OCT-A advantages}

OCT-A is a fast, non-contact and dye free imaging technique for retinal vasculature. It is an excellent option for patients requiring frequent retinal imaging. OCT-A offers quantitative

\subsection{OCT-A disadvantages}

Although this technique is safer as regards not using a fluorescein dye, yet there is lack of data offered by dye leakage in cases of inflammation. It imaging: fluorescein angiography and optical coherence tomography. OCT-A has offered a superior advantage of monitoring retinal capillaries bypassing the use of dye and overcoming the factor of time [2].

retinal location successively separated by a very brief period of time. When light is reflected back, a difference in signal will be captured between the two scans which is called the decorrelation signal. This is attributed to the movement of blood through the retinal vessels [2].

assessment of the retinal vessels through segmentation of layers with histological like resolution. In addition the deep retinal plexus and choroidal vessels are more visible using OCT-A [3,4].

also cannot give a sense of vascular filling or the filling of the peripheral vessels. As multiple devices are present in the market; each with its own 
algorithm and software, which makes interchangeability between devices not possible. In addition this applies to the

\section{Interpretation Guide to OCT-A}

To avoid misinterpretation of images: Three images should be simultaneously examined: the en face OCT-A image; the corresponding B-scan with displayed flow overlay and segmentation lines; and the structural en face intensity image for the slab at concern, fig. (1) More information is gathered from OCT-A when the signal strength is high. The signal is defined as the information derived from the tissue being imaged. Noise is the un-useful information generated during image acquisition. Cataract is one example of low image quality due to decrease in signal levels. This results in decrease in signal-to-noise ratio and hence affecting image quality. Media opacities cannot be overcome unlike dry-eyes and patient positioning which can be modified to acquire better image quality. In each commercial device, the signal strength is mentioned to judge the quality of the acquisition. There are a number of artifacts that can degrade the strength of the signal and cause misinterpretation. Bulk Motion artifact

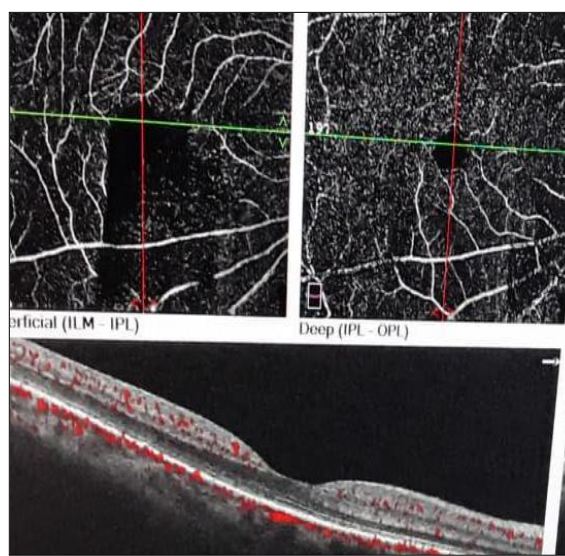

Figure 1: Normal OCT angiography

\section{How to read an OCT-A report?}

Step 1: Make sure to image the correct diseased area. Use a small scan size if the lesion is concise as macular choroidal neovascularization. Increase the scan size if used to access proliferative diabetic retinopathy. Move the internal scan size $(3 * 3$ or $6 * 6)$ which cannot be used interchangeably [5].

results from patient head movement during image session. The artifact results from misinterpreting the head movement as movement of the retinal flow. This is overcome by mechanical eye tracking or software motion correction. While shadowing artifact occurs when something like drusen or hemorrhage blocks the OCT beam [4]. One of the most common artifacts is projection artifact which results from light reflected from the retinal pigment epithelium (RPE) that is affected by the superficial capillary layers. This generates a decorrelation signal that mimics superficial vascular beds. Projection artifact is overcome by slab-subtraction of superficial retinal layers from deep layers. Segmentation artifact arises from the fact that device manufacturers constructed slab segmentation based on healthy retinal; which is not the case in pathological retina that ends up mislabeling segmentation. Correcting segmentation artifact is done through slab selection and manual segmentation [6].

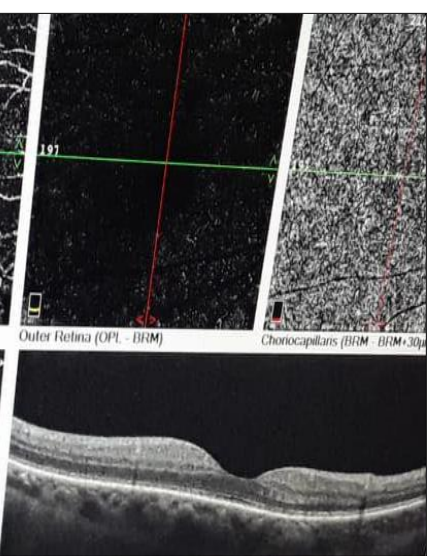

fixation target or use the external fixation light to image peripheral lesions outside macular scan as choroidal hemangioma [7]. Step 2: individual slab examination. Superficial slab spans from the internal limiting membrane, the 
ganglion cell and the inner plexiform layer. This slab usually contains the superficial capillary plexus and delineates the foveal avascular zone. Examination of this slab is beneficial in retinal diseases causing capillary malformation or drop out as diabetic retinopathy. The deep retinal slab spans the inner nuclear layer and the outer plexiform layer and includes the intermediate and deep capillary plexuses. The avascular or outer retina slab spans the outer plexiform layer and the outer nuclear layer. This

\section{Applications of OCT-A}

OCT-A in diabetic retinopathy (DR); OCT-a is superior to FA in detecting vascular changes even before occurrence of signs of retinopathy [8,9].

\subsection{Evaluation of OCT-A in DR Involves}

1-FAZ enlargement increases with the severity of DR. It should be evaluated in the full retinal depth projection to avoid artifacts [10]. 2-Microaneurysms (MA) can be monitored by OCTA but $60 \%$ lesser than FA can. This is due to the fact that MA is capillary pouches where blood flow slows down causing it not to be detectable by OCT-

5.2. OCT-A in ARMD and macular

OCT-A is an indispensable tool for detecting all structural types of MNV, fig. (2). MNV falls into three categories: MV1 that arises under the RPE. MVD 2 which is between the RPE and the retina and MVD 3 which arises from the superficial layers of the retina [16].

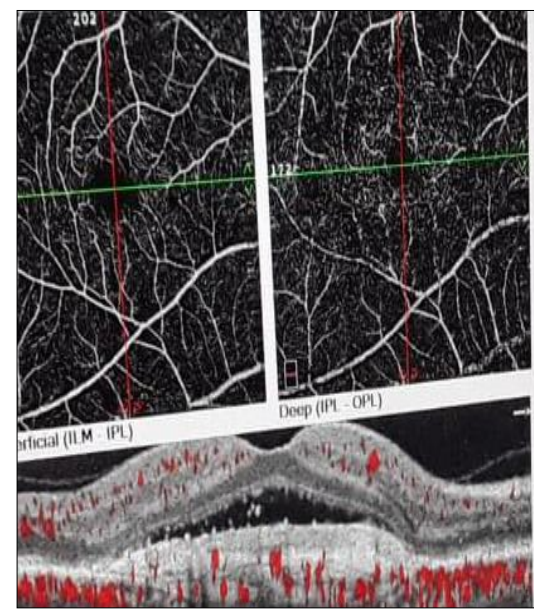

Figure 2: OCT-A in MNV. slab is avascular in healthy individuals. In age related macular degeneration, this layer may show neovascularization. Step 3: Check segmentation accuracy. OCT-A devices generate automated segmentations of slabs which are accurate in healthy retina. Incorrect slab segmentation placement results in missing information or misinterpretation of data. Step 4: B-scan image correlation with en face images In order to avoid projection artifacts.

So OCT-A can function as a screening tool as well as a follow up tool in diabetic patients.

A. ${ }^{1}$ Increasing MA and ischaemia can be prognostic of diabetic macular oedema recurrence [11]. 3-Vascular changes: Vessel density in inversely related to the severity of DR in both superficial and deep capillary plexuses [12-14]. In addition OCT-A can detect neovascularization in proliferative DR and follow its course after anti-VEGF [15].

\section{neovascularization (MNV)}

OCT-A gives information in cases of non-exudative MNV which could have been overlooked in OCT where they appear as double layer sign "flat irregular PED" [17]. Yet MNV visualization can be greatly affected by hemorrhages and large PEDs [18].

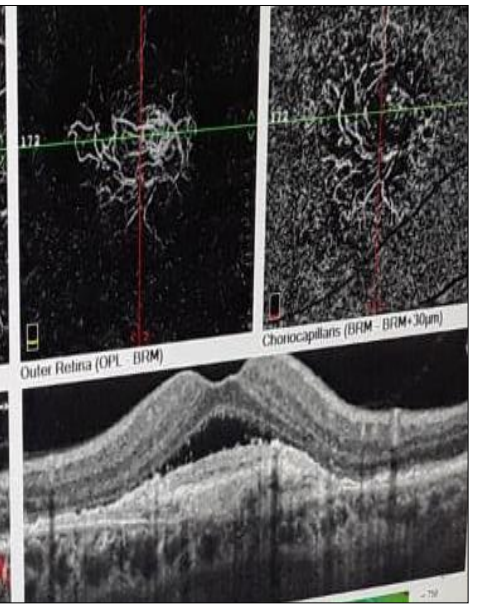




\subsection{OCT-A in other retinal disorders}

In retinal vein occlusion, OCT-A can detect areas of non-perfusion as well as areas of collaterals $[19,20]$. In addition , OCT-A can detect vasscular changes associated with inflammation or with occlusive vasculitis. ${ }^{21,22}$

\section{Conclusion}

OCT-A is a rapidly evolving technology which would make retinal diseases easier to understand and interpret. Yet full knowledge of the artifacts is mandatory to avoid any misdiagnosis. OCT-A has opened new horizons for imaging of retinal diseases and it still has not reached its full potential.

\section{References}

1. Spaide, R., Fujimoto, J., Waheed, N., et al. Optical coherence tomography angiography. Prog Retin Eye Res. 2018; 64: 1-55.

2. Greig, E., Duker, J. \& Waheed, N. A practical guide to optical coherence tomography angiography interpretation. Int. J. Retina Vitreous. 2020; 6 (1): 55.

3. Matsunaga, D., Yi, J., Puliafito, C., et al. OCT angiography in healthy human subjects. Ophthalmic Surg Lasers Imaging Retina. 2014; 45 (6): 510-515.

4. Borrelli, E., Sarraf, D., Freund, K., et al. OCT angiography and evaluation of the choroid and choroidal vascular disorders. Prog Retin Eye Res. 2018; 67: 30-55.

5. Rabiolo, A., Gelormini, F., Marchese, A., et al. Macular perfusion parameters in different angiocube sizes: Does the size matter in quantitative optical coherence tomography angiography? Invest Ophthalmol Vis Sci.; 59 (1): 231237.

6. Spaide, R., Fujimoto, J., Waheed, N. Image artifacts in optical coherence tomography angiography. Retina. 2016; 44 (5): 367-368.

7. Callaway, N., Mruthyunjaya, P. Widefield imaging of retinal and choroidal tumors. Int. J. Retina Vitreous. 2019; 5 (Suppl 1): 49.

8. Yasin Alibhai, A., Moult, E., Shahzad, R., et al. Quantifying microvascular changes using OCT angiography in diabetic eyes without clinical evidence of retinopathy. Ophthalmol Retina. 2018; 2: 418-427.
9. Takase, N., Kato, A., Ozeki, H., et al. Enlargement of foveal avascular zone in diabetic eyes evaluated by en face optical coherence tomography angiography. Retina. 2015; 35: 2377 2383.

10. Bresnick, G., Condit, R., Syrjala, S., et al. Abnormalities of the foveal avascular zone in diabetic retinopathy. Arch Ophthalmol. 1984; 102 (9): 1286-1293.

11. Hasegawa, N., Nozaki, M., Takase, $\mathrm{N}$., et al. New insights into micro aneurysms in the deep capillary plexus detected by optical coherence tomography angiography in diabetic macular edema. Invest Ophthalmol Vis Sci. 2016; 57 (9): OCT 348- OCT 355.

12. Sambhav, K., Abu-Amero, K., Chalam, K. Deep capillary macular perfusion indices obtained with OCT angiography correlate with degree of nonproliferative diabetic retinopathy. Eur J. Ophthalmol. 2017; 27 (6): 716-729.

13. Durbin, M., An, L., Shemonski, N., et al. Quantification of retinal microvascular density in optical coherence tomographic angiography images in diabetic retinopathy. JAMA Ophthalmol. 2017; 135 (4): 370-376.

14. Nesper, P., Roberts, P., Onishi, A., et al. Quantifying microvascular abnormalities with increasing severity of diabetic retinopathy using optical coherence tomography angiography. Invest Ophthalmol Vis Sci. 2017; 58 (6): BIO307-BIO3015.

15. Hu, Z., Su, Y., Xie, P., et al. OCT angiography-based monitoring of neovascular regression on fibrovascular 
membrane after preoperative intravitreal conbercept injection. Graefes Arch Clin Exp Ophthalmol. 2019; 257 (8): 1611-1619.

16. Freund, K. Zweifel, S, Engelbert, M. Do we need a new classification for choroidal neovascularization in agerelated macular degeneration? Retina. 2010; 30: 1333-1349.

17. Carnevali, A., Cicinelli, M., Capuano, V., et al. Optical coherence tomography angiography: A useful tool for diagnosis of treatment-naive quiescent choroidal neovascularization. Am. J. Ophthalmol. 2016; 169: 189-198.

18. De Carlo, T., Bonini Filhom M., Chin, A., et al. Spectral-domain optical coherence tomography angiography of choroidal neovascularization. $\boldsymbol{O p h}$ thalmology. 2015; 122: 1228-1238.
19. Suzuki, N., Hirano, Y., Tomiyasu, T., et al. Collateral vessels on optical coherence tomography angiography in eyes with branch retinal vein occlusion. Br. J. Ophthalmol. 2019, 103 (10): 1373-1379.

20. Novias, E. \& Waheed, N. Optical coherence tomography angiography of retinal vein occlusion. Dev. Ophthalmol. 2016; 56: 132-138

21. Pichi, F., Sarraf, D., Arepalli, S., et al. The application of optical coherence tomography angiography in uveitis and inflammatory eye diseases. Prog Retin Eye Res. 2017; 59: 178-201.

22. Anvernizzi, A., Cozzi M., Staurenghi. G. Optical coherence tomography and optical coherence tomography angiography in uveitis: A review. Clin Exp Oph-thalmol. 2019; 47 (3): 357-371. 